\title{
Memory Modulation Across Neural Systems: Intra-Amygdala Glucose Reverses Deficits Caused by Intraseptal Morphine on a Spatial Task But Not on an Aversive Task
}

\author{
Ewan C. McNay and Paul E. Gold \\ Neuroscience Program and Department of Psychology, University of Virginia, Charlottesville, Virginia 22903
}

\begin{abstract}
Based largely on dissociations of the effects of different lesions on learning and memory, memories for different attributes appear to be organized in independent neural systems. Results obtained with direct injections of drugs into one brain region at a time support a similar conclusion. The present experiments investigated the effects of simultaneous pharmacological manipulation of two neural systems, the amygdala and the septohippocampal system, to examine possible interactions of memory modulation across systems. Morphine injected into the medial septum impaired memory both for avoidance training and during spontaneous alternation. When glucose was concomitantly administered to the amygdala, glucose reversed the morphine-induced deficits in memory during alternation but not
\end{abstract}

for avoidance training. These results suggest that the amygdala is involved in modulation of spatial memory processes and that direct injections of memory-modulating drugs into the amygdala do not always modulate memory for aversive events. These findings are contrary to predictions from the findings of lesion studies and of studies using direct injections of drugs into single brain areas. Thus, the independence of neural systems responsible for processing different classes of memory is less clear than implied by studies using lesions or injections of drugs into single brain areas.

Key words: glucose; memory; neural systems; medial septum; amygdala; morphine; spontaneous alternation; inhibitory avoidance
Experiments in which lesions of different brain areas impair performance on specific tasks support the common suggestion that the neural substrates of learning and memory are organized into multiple distinct neural systems. For example, lesions of the septohippocampal system impair memory for tasks dependent on spatial, contextual, or relational features (O'Keefe and Nadel, 1978; Squire and Zola-Morgan, 1991; Eichenbaum, 1992; Shen et al., 1996); lesions of the neostriatum impair memory for tasks dependent on cues or responses (Packard et al., 1989; Packard and McGaugh, 1992; Kesner et al., 1993; Oliveira et al., 1997); and lesions of the amygdala impair memory for tasks dependent on affective information (McGaugh et al., 1992; LeDoux, 1993; Maren and Fanselow, 1996; Davis, 1997). With appropriately selected tasks and neural systems, the effects of brain lesions show double and triple dissociations across tasks (Packard et al., 1989; Kesner et al., 1993; McDonald and White, 1993; Bechara et al., 1995), suggesting that the lesioned areas belong to and participate in only one of several memory systems.

Drug administration to single brain areas gives results consistent with expectations based on lesion analyses, with effects seen only on performance of those tasks affected by lesion of the brain area (McGaugh et al., 1993; for review, see Gold, 1995). For example, injection of morphine into the medial septum causes deficits in memory for tasks with spatial components, such as spontaneous alternation and inhibitory avoidance, and glucose

Received Dec. 19, 1997; revised Feb. 18, 1998; accepted Feb. 26, 1998.

This work was supported by National Institute on Aging Grant AG 07648 and National Institute of Neurological Diseases and Stroke Grant NS 32914. We thank Ray Kesner for his thoughtful comments on the results.

Correspondence should be addressed to Ewan C. McNay, Department of Psychology, 102 Gilmer Hall, University of Virginia, Charlottesville, VA 22903. Electronic mail may be sent to ewan@virginia.edu.

Copyright (C) 1998 Society for Neuroscience $0270-6474 / 98 / 183853-06 \$ 05.00 / 0$ co-administration into the same area reverses these deficits (Ragozzino et al., 1992). However, the same drug injections into the amygdala modulate learning and memory only for an affective inhibitory avoidance task, not for spontaneous alternation (Ragozzino and Gold, 1994).

These results of administration of morphine and glucose to distinct brain areas suggested that concurrent pharmacological manipulation of the medial septum and amygdala might permit investigation of interactions of memory modulators across putatively distinct neural systems. In the present experiments, morphine was injected into the medial septum, causing deficits in both spontaneous alternation performance and inhibitory avoidance learning while glucose was co-administered into the amygdala. The design pitted two hypotheses against each other. If the amygdala and septohippocampal system are responsible for different attributes of memory and are part of distinct neural systems, then the two manipulations should not interact; deficits caused by morphine should not be attenuated by glucose injections into the amygdala. If the amygdala is either a primary anatomical substrate for affective memories (LeDoux, 1993; Davis, 1997) or a site of modulation of memory processes during emotional arousal (McGaugh et al., 1992, 1996; Roozendaal and McGaugh, 1997), then glucose injections into the amygdala should selectively attenuate deficits in the aversive avoidance task but not the alternation task. Surprisingly, the results supported neither hypothesis. Intra-amygdala injections of glucose reversed the impairments produced by intraseptal injections of morphine on the spontaneous alternation task but not the inhibitory avoidance task.

\section{EXPERIMENT 1: INHIBITORY AVOIDANCE}

Administration of morphine to either the medial septum or amygdala causes deficits in memory for inhibitory avoidance training. 
Figure 1. Location of injection cannula tips and diffusion of ink in the medial septum (left) and amygdala (right) for all rats included in analyses. Rat brain sections were taken from the atlas of Pellegrino et al. (1979).

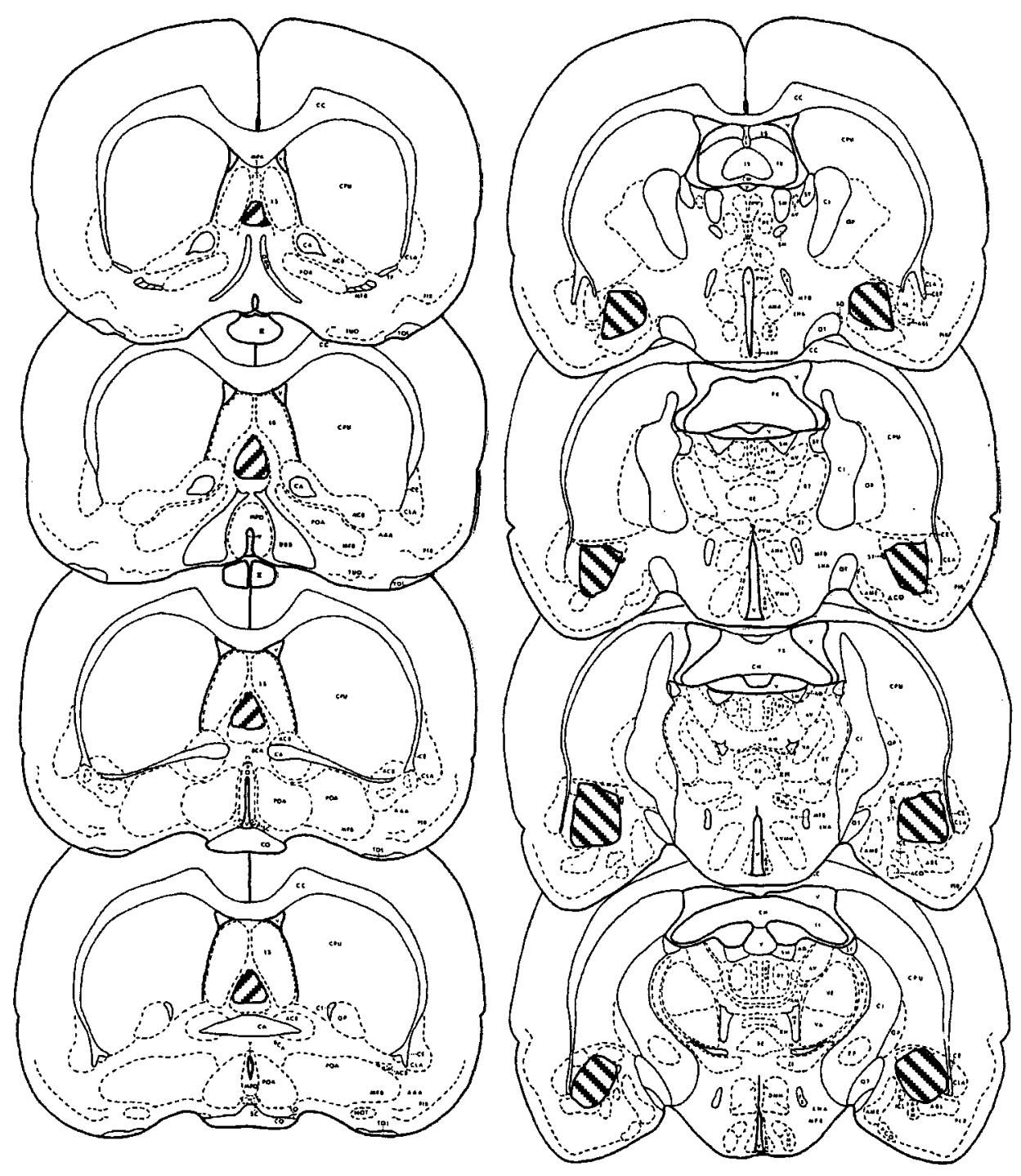

Administration of glucose alone peripherally or to either brain area has no effect on performance, but concurrent administration of glucose to the same brain area as morphine reverses the morphine-induced deficits (Ragozzino et al., 1992; Ragozzino and Gold, 1994). Thus, this task was chosen as one likely to permit demonstration of an interaction of the two modulators across neural systems.

\section{Materials and Methods}

Subjects. Male Sprague Dawley rats (Charles River, Wilmington, MA), 3 months old at time of surgery, were used as subjects. Rats were individually housed with food and water available ad libitum and were maintained on a $12 \mathrm{hr}$ light/dark schedule (lights on at 7:00 A.M.).

Surgery. Rats received atropine sulfate $(0.2 \mathrm{cc}$ of a $540 \mathrm{mg} / \mathrm{cc}$ solution, i.p.) $10 \mathrm{~min}$ before being anesthetized with sodium pentobarbital $(50$ $\mathrm{mg} / \mathrm{kg}$, i.p.). Standard sterile stereotaxic procedures were used to implant stainless steel guide cannulae (22 ga; Plastics One, Inc., Roanoke, VA) into the medial septum and amygdala. Three cannulae were implanted in each rat: one aimed at the medial septum and two bilaterally aimed dorsal to the central nucleus of the amygdala. The nose bar was set at $5.0 \mathrm{~mm}$ above the interaural line according to the atlas of Pellegrino et al. (1979), and coordinates were $1.3 \mathrm{~mm}$ anterior to bregma, 0.0 lateral and 4.5 ventral from dura (medial septum), and $0.3 \mathrm{~mm}$ anterior to bregma, \pm 4.6 lateral and 7.0 ventral from dura (amygdala). Stylets, constructed from 28 ga injection cannulae cut to the length of the implanted cannulae, were inserted into all implanted cannulae to main- tain patency. Rats received injections of sterile saline $(6 \mathrm{cc}$, s.c.) and were then placed in a warm incubator until they had recovered from anesthesia. Rats were allowed to recover for 1 week before behavioral testing. During the recovery period, all animals were handled individually for a minimum of 5 min each day.

Behavioral procedures. Inhibitory avoidance training was conducted in a trough-shaped alley $(90 \mathrm{~cm}$ long, $15 \mathrm{~cm}$ high, $5.5 \mathrm{~cm}$ wide at floor, and $20 \mathrm{~cm}$ wide at ceiling) divided into two compartments by a sliding door: a well lit white compartment $30 \mathrm{~cm}$ long, and a dark compartment $60 \mathrm{~cm}$ long. Both compartments were covered by a black Plexiglas ceiling. On the training trial, rats were placed into the well lit compartment. After a delay of $10 \mathrm{sec}$, the sliding door was retracted, and the rat was allowed to enter the dark compartment. Immediately after entry into this dark compartment, a brief foot shock $(1.0 \mathrm{~mA}, 1 \mathrm{sec})$ was administered through metal plates constituting the floor and walls of the dark compartment. Upon being shocked, rats returned rapidly to the well lit compartment and were then immediately removed and returned to their home cage. Retention was measured $24 \mathrm{hr}$ later by placing each rat into the well lit compartment. After a delay of $10 \mathrm{sec}$, the sliding door was again retracted, and the latency to enter the dark compartment was recorded (600 sec maximum).

Intracranial injections. Injections were administered to both the medial septum and bilaterally to the amygdala through an injection cannula that extended $1.0 \mathrm{~mm}$ (medial septum) or $1.5 \mathrm{~mm}$ (amygdala) below the tip of the guide cannula. The 28 ga injection cannula was attached to a $25 \mu \mathrm{l}$ Hamilton syringe via polyethylene tubing, and the tubing was attached to an infusion pump (Harvard Apparatus). All solutions were delivered in 


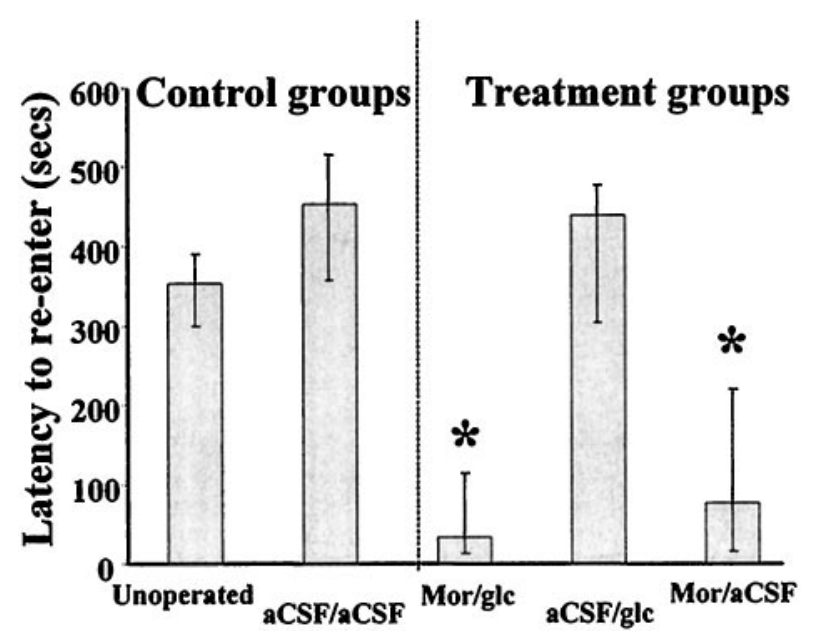

Figure 2. Median latencies to reenter the inhibitory avoidance chamber $24 \mathrm{hr}$ after training. Error bars indicate interquartile ranges. Mor, Animals receiving morphine into the medial septum; $g l c$, animals receiving glucose into the amygdala; $a C S F$, animals receiving artificial CSF into the medial septum and/or the amygdala. Asterisk indicates significantly lower median latency than unoperated, vehicle, and glucose-only groups; $p<0.01$.

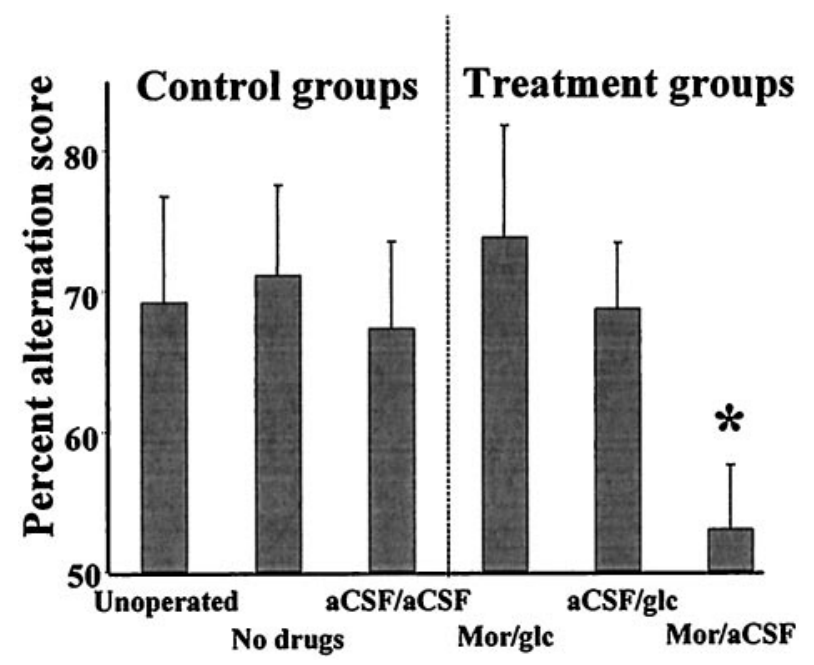

Figure 3. Mean alternation scores in Y maze expressed as a percentage of possible alternations (consecutive entry into each of the three arms without repetition). Error bars indicate SE. No drugs, Animals with implanted cannulae but receiving no injections. Other groups are the same as in Figure 2. Asterisk indicates significantly lower mean alternation score versus all other groups; $p<0.01$.

a volume of $0.5 \mu \mathrm{l}$ at a rate of $0.25 \mu \mathrm{l} / \mathrm{min}$. Before removal, the injection cannula was left in place for an additional minute to facilitate drug diffusion. All drugs were mixed in artificial CSF (aCSF, pH 7.4) that contained $3.3 \mathrm{~mm}$ glucose.

Rats in experimental groups received intracranial injections $30 \mathrm{~min}$ before training. This timing was chosen to be the same used in previous studies (Ragozzino et al., 1992; Ragozzino and Gold, 1994) and also to permit identical manipulations of memory in the two experiments (because post-training injections would not be possible in Experiment 2). Rats were assigned randomly to one of four groups: (1) aCSF and aCSF $(n=7)$; rats received injections of vehicle (aCSF) into both the medial septum and bilaterally into the amygdala; (2) morphine and aCSF $(n=$ 11); rats received injections of $4.0 \mathrm{nmol}$ of morphine sulfate into the medial septum and of vehicle into both amygdala; (3) aCSF and glucose $(n=6)$; rats received injections of vehicle into the medial septum and $16.7 \mathrm{nmol}$ of glucose into both amygdalae; and (4) morphine and glucose $(n=9)$; rats received injections of $4.0 \mathrm{nmol}$ of morphine sulfate into the medial septum and $16.7 \mathrm{nmol}$ of glucose into both amygdalae. The doses

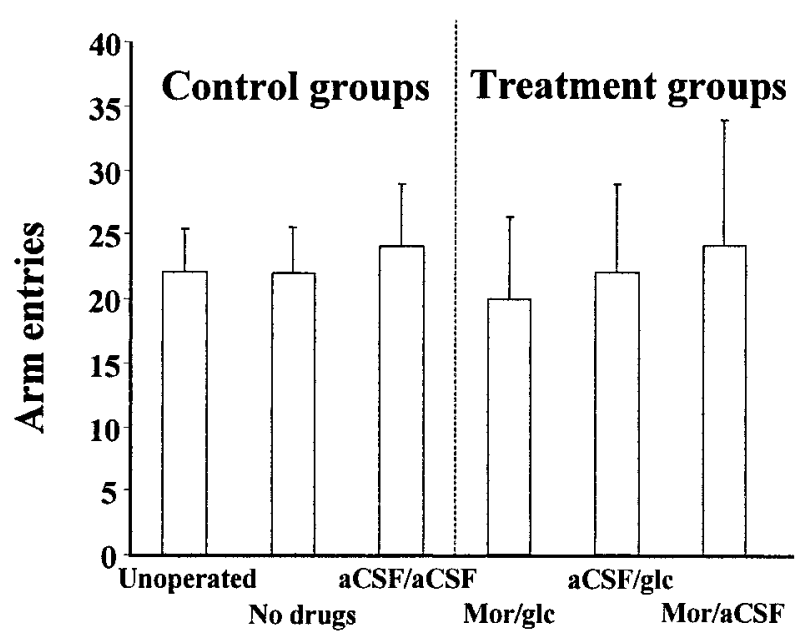

Figure 4. Mean number of arm entries during Y maze alternation task. Error bars indicate SE. Drug groups are the same as in Figure 3.

of morphine and glucose used were chosen based on previous findings indicating that these doses were effective in modulating memory test performance in these brain areas and did not alter flinch-jump thresholds to foot shock when administered to the amygdala (Ragozzino et al., 1992; Ragozzino and Gold, 1994). An unoperated control group (group $5 ; n=7$ ) received no injections but was otherwise handled identically to the experimental groups.

Histology. After completion of testing, rats were killed by overdose of sodium pentobarbital. Ink $(0.25 \mu \mathrm{l})$ was injected into each cannula by means of an injection cannula to aid in histological verification. Intracardial perfusions were performed with $0.9 \%$ saline followed by a $10 \%$ formalin solution. Brains were removed and placed in a $30 \%$ sucrose/ $10 \%$ formalin solution for a minimum of $3 \mathrm{~d}$. Before sectioning, brains were frozen at $-20^{\circ} \mathrm{C}$ and mounted on a Reichert-Jung cryostat. Sections $(40 \mu \mathrm{m})$ were taken through the brain areas of cannula placement, mounted onto slides, dried, and stained with cresyl violet. Figure 1 illustrates acceptable placement locations for tips of injection cannulae and ink diffusion into the medial septum and amygdala. Data from 20 animals (in addition to the $n$ values given above) were discarded because of misplacement of one or more cannulae.

Statistical analysis. Because the distribution of latencies was truncated by the $600 \mathrm{sec}$ maximum, the results were analyzed with nonparametric Mann-Whitney $U$ tests (two-tailed).

\section{Results}

As shown in Figure 2, rats that received vehicle into the medial septum and bilaterally into the amygdala had retention latencies that did not differ from those of unoperated control animals $\left(U_{(7,7)}=12 ; p>0.1\right)$. Similarly, administration of glucose into the amygdala did not significantly alter latencies compared with administration of vehicle alone $\left(U_{(6,7)}=18 ; p>0.7\right)$. Rats receiving morphine into the medial septum showed significant reductions in latency compared with rats in either vehicle or implanted control groups $\left(U_{(11,7)}=7\right.$ and 9 , respectively; $\left.p<0.01\right)$. Concurrent infusion of glucose into the amygdala did not reduce the effect of morphine administration; rats that received both intraseptal morphine and intra-amygdala glucose had latencies comparable to those of rats that received morphine alone $\left(U_{(11,9)}=39 ; p>0.4\right)$ and significantly below those of rats in the vehicle and control groups $\left(U_{(9,7)}=0\right.$ and 4 , respectively; $p<0.002$ and 0.01 , respectively). Initial latencies to enter the dark compartment on the training trial did not differ across groups $(p>0.3)$.

\section{EXPERIMENT 2: SPONTANEOUS ALTERNATION}

The results of Experiment 1 show that glucose administration into the amygdala does not reverse deficits in aversive memory 
caused by septal morphine administration, in contrast to the results obtained by co-administering the two drugs to the same brain area (Ragozzino et al., 1992; Ragozzino and Gold, 1994). Although many past experiments have demonstrated a role for the amygdala in affective memory, manipulations of the amygdala have consistently failed to affect learning or memory for spatial attributes as assessed by, for example, alternation tasks (Ragozzino and Gold, 1994; Wan et al., 1994; Lennartz et al., 1996). It therefore seemed likely that glucose injections into the amygdala would also fail to reverse the deficits produced by intraseptal morphine injections on a nonaffective spontaneous alternation task.

\section{Materials and Methods}

Behavioral procedures. One week after inhibitory avoidance testing, the same rats used in Experiment 1 were tested in a Y maze for spontaneous alternation behavior. The rats were handled daily for $5 \mathrm{~min}$ during this week. Cannulated rats (i.e., all groups except unoperated controls) were randomly reassigned to a drug group with the restriction that no rat could be placed in the same group as that to which it had belonged for inhibitory avoidance testing. The same treatment and control groups were run as in Experiment 1. In addition, an extra control group (group 6 , operated controls that were subject to the same surgical procedures as the experimental groups but received no injections) was added. The purpose of this group was to confirm that neither damage caused by injection cannulae as part of the inhibitory avoidance experiment nor exposure to the inhibitory avoidance task affected performance on this second task. Groups in this experiment had $n$ values of $8,8,9,8,7$, and 5 , respectively.

The measure of spatial working memory taken was percent alternation, as previously used in our laboratory and by others (Sarter et al., 1988; Beracochea and Jaffard, 1990; Ragozzino et al., 1992; Ragozzino and Gold, 1994) (for review, see Dember, 1989). Briefly, this is the number of possible complete alternations (nonrepeating choices of each of the three maze arms) as a percentage of the total number of possible alternations (number of arm choices made minus 2). The total number of arm entries made was also recorded for use as a measure of locomotor activity. The $\mathrm{Y}$ maze was composed of three troughs radiating from a triangular central area. Each arm measured $60 \mathrm{~cm}$ long $\times 17.5 \mathrm{~cm}$ high, with a width at floor of $3.5 \mathrm{~cm}$ and at ceiling of $14 \mathrm{~cm}$. The central area was thus an equilateral triangle with $3.5 \mathrm{~cm}$ sides at floor. All arms and the central area were covered by a darkly tinted Plexiglas ceiling.

Statistical analysis. Spontaneous alternation scores and arm entries were analyzed by independent two-tailed $t$ tests.

\section{Results}

Rats that received morphine injections into the medial septum had alternation scores significantly lower than those that received vehicle injections, and also lower than those of both operated and unoperated control animals ( $p<0.001$ vs vehicle; $p<0.01$ vs operated and unoperated control groups). Concurrent inf usion of glucose into the bilateral amygdala of animals receiving septal morphine produced alternation scores comparable to those exhibited by control rats and rats receiving only vehicle, as shown in Figure $3(p>0.1)$. Glucose administration into the amygdala alone had no effect on alternation performance; animals receiving only glucose were again not different from either vehicle or uninjected control groups $(p>0.3)$.

As shown in Figure 4, neither drug treatments nor surgery alone had any effect on locomotor activity as measured by number of arm choices made $(p>0.05)$.

\section{DISCUSSION}

Consistent with past findings (Ragozzino et al., 1992; Ragozzino and Gold, 1994), administration of morphine into the medial septum impaired spontaneous alternation performance and memory for inhibitory avoidance training. This finding is also consistent with results from studies using either septal lesions or a variety of pharmacological manipulations in which effects on both inhibitory avoidance and spatial memory tasks have been demonstrated (Capobianco et al., 1977; Gittis and Gordon, 1977; Bartsch and Enloe, 1978; Givens and Olton, 1995; Wan et al., 1995).

The main new findings in the present experiments are that glucose injections into the amygdala reverse the impairments produced by intraseptal morphine in spontaneous alternation but not in inhibitory avoidance. These findings run contrary to predictions from experiments using either lesions or manipulations of the amygdala in which effects are seen on affective memory but not on spatial memory, including spontaneous alternation performance (Aggleton et al., 1989; Ragozzino and Gold, 1994; Wan et al., 1994).

In Experiment 1, intra-amygdala glucose administration failed to attenuate the impairments induced by intraseptal morphine in memory for inhibitory avoidance training. The failure to attenuate contrasts with the full reversal of deficits seen when glucose is co-injected with morphine into either the medial septum or amygdala using identical doses of drugs to those used here. On the basis of the results of this experiment alone, one might suggest either that the neural systems are indeed independent or that manipulations of the medial septum are dominant over those of the amygdala. However, the results of Experiment 2 showed that neither of these is true.

Given the results of Experiment 1, we hypothesized in Experiment 2 that intra-amygdala glucose injections would not attenuate the impairments induced by intraseptal morphine injections in spontaneous alternation performance. This hypothesis was proven incorrect; intra-amygdala glucose fully reversed the morphine-induced impairments on this spatial working memory task. The finding that administration of glucose to the amygdala reverses the effect of septal morphine on memory in this task shows that interactions in modulation of memory are not limited to those within a single brain area. These results represent the first demonstration, as far as we are aware, of amygdala involvement in modulation of memory processes for a nonaffective task [although there have been previous reports of amygdala involvement in various aversively motivated water maze tasks (Packard et al., 1994; Roozendaal et al., 1996; Roozendaal and McGaugh, 1997)]. Several previous experimenters, using either lesions or pharmacological manipulations within an area, have concluded that the amygdala appears to play no role in memory or in modulation of memory for spatial information (Kesner, 1992; McDonald and White, 1993, 1995; Ragozzino and Gold, 1994). Evidently, this is not always the case. Taken alone, the results of this experiment would be consistent with the view that the amygdala is able to modulate memory processes in many different systems, a hypothesis similar to that of McGaugh et al. (1992, 1996) but extended to all tasks rather than just those involving emotional arousal. However, the inhibitory avoidance findings of Experiment 1 do not permit this more generalized conclusion.

Considered together, the results of Experiments 1 and 2 present a picture that is both complex and surprising. The surprise can perhaps best be seen if one imagines how these results would be interpreted if there was no previous work on involvement of neural systems in different types of memory. If that were the case, then on the basis of the data shown here one might conclude that although the septohippocampal system is involved in both spatial and aversive memory processes, the amygdala is specifically involved in spatial processes and not in aversive memory. Such a conclusion would be in direct opposition to the several 
demonstrations of dissociations in which the amygdala appears to be involved in aversive memory but not in performance on spatial tasks (Packard and White, 1991; Kesner et al., 1993; McDonald and White, 1993; Ragozzino and Gold, 1994; Wan et al., 1994; Lennartz et al., 1996).

It is of course possible that there exists a dose of glucose that could be administered to the amygdala to reverse the effects of septal morphine on avoidance learning. However, this seems unlikely; the dose of glucose used is effective in reversing the effects of morphine within both the amygdala and the septum on both of the tasks used here (Ragozzino et al., 1992; Ragozzino and Gold, 1994). Furthermore, the results of Experiment 2 show this dose to be effective in reversing the effects of morphine across brain areas in at least one task. Not only does the same dose of glucose reverse the effects of morphine on memory in both tasks within both brain areas, but a single systemic dose of glucose also reverses the effects of septal morphine on both tasks (Ragozzino et al., 1992). Finally, it is worth noting that the aCSF used in the present experiments contains a level of glucose $(3.3 \mathrm{~mm})$ that is $\sim 300 \%$ of that found in the rat hippocampal extracellular fluid at rest (McNay and Gold, 1997), so that it could be considered a second lower dose that also fails to reverse the effects of morphine on the avoidance task.

The present results are clearly not consistent with the idea that the amygdala and septohippocampal system are involved only in separate memory systems, as suggested by data from studies using lesions and/or pharmacological manipulations of single brain areas. Rather, they suggest that areas of the brain responsible for memory processes are linked and inter-related. The separate neural systems proposed to underlie different types of memory on the basis of dissociation studies may well be different components of a larger integrated system. This suggestion is supported by recent experiments which have begun to investigate the interactions of lesions and pharmacological manipulations of separate brain areas (Roozendaal and McGaugh, 1996, 1997).

McDonald and White (1995) suggested that the hippocampus might play the dominant role in any hierarchy of neural systems of memory, but the results of Experiment 2 in which a manipulation of the amygdala reverses the effects of a septohippocampal manipulation suggest a more complex arrangement. Moreover, our results show that brain areas involved in memory, as well as modulators of memory, may interact in ways not readily predictable from manipulations within any one brain area. In contrast to lesion experiments in which one or more brain areas are removed and unavailable for use or manipulation, examinations of interactions across memory systems are readily enabled by using direct manipulation of two or more intact neural systems.

One explanation for the present results might be that the amygdala is able to enhance memory processes only under conditions of arousal and that the administration of glucose to the amygdala in Experiment 2 creates (one or more of) the physiological consequences of arousal where none was previously present. Under this hypothesis, because the inhibitory avoidance task in Experiment 1 involves an effective level of arousal as part of the training, simulation of further arousal would not enhance memory formation. A slightly different possibility is that the amygdala is not normally involved in mediating the spatial memory processes required in spontaneous alternation but is recruited to do so by administration of glucose and takes the place of the impaired septal mediation. Because amygdala processes are involved normally in inhibitory avoidance, administration of glucose to the amygdala in this task is unable to recruit it to overcome the deficits caused by septal morphine. If arousal or affective content of a task is the factor that determines whether the amygdala is active in processing memory for that task, then the two hypotheses converge. Such a hypothesis is consistent with previous data (Gold et al., 1975, 1978) showing that (1) stimulation of the amygdala is effective in reversing deficits attributable to amygdala lesions in a low-shock task (when stimulation would be hypothesized to recruit other brain areas); and (2) identical stimulation causes a deficit in the animals with the same lesion on a high-shock task in which no deficit was found to be attributable to lesion alone [when high-shock would be hypothesized to have already recruited alternative brain areas, and further stimulation would push activation onto the down-slope of the inverted-U response curve generally seen in memory modulation (Koob, 1991)]. Similarly, this hypothesis explains the finding that manipulations of the amygdala can affect memory for many attributes of aversive tasks, including some attributes that may be nonaversive (Packard et al., 1994; Roozendaal et al., 1996; Roozendaal and McGaugh, 1997). Furthermore, this hypothesis is consistent with the proposal of McGaugh et al. $(1992,1996)$ that the amygdala modulates memory processes, but it changes the proposal to say that the amygdala may modulate memory processes not only at times of emotional arousal, but generally.

In summary, the present data show that injections of drugs into two brain areas traditionally believed to belong to separate neural systems can interact to modulate memory processes, and that such interactions are not predictable on the basis of either lesion data or findings from drug administration to single brain areas.

\section{REFERENCES}

Aggleton JP, Blindt HS, Rawlins JN (1989) Effects of amygdaloid and amygdaloid-hippocampal lesions on object recognition and spatial working memory in rats. Behav Neurosci 103:962-974.

Bartsch DA, Enloe LJ (1978) Behavioral differentiation of septal lesions in two strains of rats. Physiol Psychol 6:478-484.

Bechara A, Tranel D, Damasio H, Adolphs R, Rockland C, Damasio AR (1995) Double dissociation of conditioning and declarative knowledge relative to the amygdala and hippocampus in humans. Science 269:1115-1118.

Beracochea DJ, Jaffard R (1990) Effects of ibotenic lesions of mammillary bodies on spontaneous and rewarded spatial alternation in mice. $\mathrm{J}$ Cognit Neurosci 2:133-140.

Capobianco S, MacDougall JM, Foster SM (1977) Direct neurobehavioral comparisons within the septohippocampal system. Physiol Psychol 5:215-220.

Davis M (1997) Neurobiology of fear responses: the role of the amygdala. J Neuropsychiatry Clin Neurosci 9:382-402.

Dember WN (1989) Spontaneous alternation behavior. New York: Springer.

Eichenbaum H (1992) The hippocampal system and declarative memory in animals. J Comp Neurol 4:217-231.

Gittis AG, Gordon M (1977) Developmental analysis of behavioral dysfunction in rats with septal lesions. J Comp Physiol Psychol 91:94-106.

Givens BS, Olton DS (1995) Bidirectional modulation of scopolamineinduced working memory impairments by muscarinic activation of the medial septal area. Neurobiol Learn Mem 63:269-276.

Gold PE (1995) Modulation of emotional and non-emotional memories: same pharmacological systems, different neuroanatomical systems. In: Brain and memory: modulation and mediation of neuroplasticity (McGaugh JL, McGaugh JL, Weinberger NM, Lynch G, eds), pp 41-74. New York: Oxford UP.

Gold PE, Hankins LL, Edwards RM, Chester J, McGaugh JL (1975) Memory interference and facilitation with posttrial stimulation: effect on memory varies with footshock level. Brain Res 86:509-513.

Gold PE, Rose RP, Hankins LL (1978) Retention impairment produced by unilateral amygdala implantation: reduction by posttrial amygdala stimulation. Behav Biol 2:515-523.

Kesner RP (1992) Learning and memory in rats with an emphasis on the role of the amygdala. In: The amygdala: neurobiological aspects of 
emotion, memory and mental dysfunction (Aggleton JP, ed), pp 379399. New York: Wiley.

Kesner RP, Bolland BL, Dakis M (1993) Memory for spatial locations, motor responses, and objects: triple dissociation among the hippocampus, caudate nucleus and extrastriate visual cortex. Exp Brain Res 93:462-470.

Koob GF (1991) Arousal, stress, and inverted U-shaped curves: implications for cognitive function. In: Perspectives on cognitive neuroscience (Lister RG, Weingartner HJ, eds) pp 300-313. New York: Oxford UP.

LeDoux JE (1993) Emotional memory systems in the brain. Behav Brain Res 58:69-79.

Lennartz RC, Hellems KL, Mook ER, Gold PE (1996) Inhibitory avoidance impairments induced by intra-amygdala propanolol are reversed by glutamate but not glucose. Behav Neurosci 110:1-7.

Maren S, Fanselow MS (1996) The amygdala and fear conditioning: has the nut been cracked? Neuron 16:237-240.

McDonald RJ, White NM (1993) A triple dissociation of memory systems: hippocampus, amygdala, and dorsal striatum. Behav Neurosci 107:3-22.

McDonald RJ, White NM (1995) Information acquired by the hippocampus interferes with acquisition of the amygdala-based conditioned-cue preference in the rat. Hippocampus 5:189-197.

McGaugh JL, Introini-Collison IB, Cahill LF, Kim M, Liang KC (1992) Involvement of the amygdala in neuromodulatory influences on memory storage. In: The amygdala: neurobiological aspects of emotion, memory and mental dysfunction (Aggleton JP, ed), pp 431-451. New York: Wiley.

McGaugh JL, Introini-Collison IB, Cahill LF, Castellano C, Dalmaz C, Parent MB, Williams CL (1993) Neuromodulatory systems and memory storage: role of the amygdala. Behav Brain Res 58:81-90.

McGaugh JL, Cahill LF, Roozendaal B (1996) Involvement of the amygdala in memory storage: interaction with other brain systems. Proc Natl Acad Sci USA 93:13508-13514.

McNay EC, Gold PE (1997) Microdialysis measurement of brain extracellular glucose concentrations: basal levels and changes during behavioral testing in awake, freely moving rats. Soc Neurosci Abstr 23:823.16.

O'Keefe J, Nadel L (1978) The hippocampus as a cognitive map. Oxford: Oxford UP.

Oliveira MGM, Bueno OFA, Pomarico AC, Gugliano EB (1997) Strategies used by hippocampal- and caudate-putamen-lesioned rats in a learning task. Neurobiol Learn Mem 68:32-41.

Packard MG, White NM (1991) Dissociation of hippocampus and caudate nucleus memory systems by posttraining intracerebral injection of dopamine agonists. Behav Neurosci 105:295-306.
Packard MG, McGaugh JL (1992) Double dissociation of fornix and caudate nucleus lesions on acquisition of two water maze tasks: further evidence for multiple memory systems. Behav Neurosci 106:439-446.

Packard MG, Hirsch R, White NW (1989) Differential effects of fornix and caudate nucleus lesions on two radial maze tasks: evidence for multiple memory systems. J Neurosci 9:1465-1472.

Packard MG, Cahill LF, McGaugh JL (1994) Amygdala modulation of hippocampal-dependent and caudate nucleus-dependent memory processes. Proc Natl Acad Sci USA 91:8477-8481.

Pellegrino LJ, Pellegrino AS, Cushman AJ (1979) A stereotaxic atlas of the rat brain. New York: Plenum.

Ragozzino ME, Gold PE (1994) Task-dependent effects of intraamygdala morphine injections: attenuation by intra-amygdala glucose injections. J Neurosci 14:7478-7485.

Ragozzino ME, Parker ME, Gold PE (1992) Spontaneous alternation and inhibitory avoidance impairments with morphine injections into the medial septum. Attenuation by glucose administration. Brain Res 597:241-249.

Roozendaal B, McGaugh JL (1996) The memory-modulatory effects of glucocorticoids depend on an intact stria terminalis. Brain Res 709:243-250.

Roozendaal B, McGaugh JL (1997) Basolateral amygdala lesions block the memory-enhancing effect of glucocorticoid administration in the dorsal hippocampus of rats. Eur J Neurosci 9:76-83.

Roozendaal B, Portillo-Marquez G, McGaugh JL (1996) Basolateral amygdala lesions block glucocorticoid-induced modulation of memory for spatial learning. Behav Neurosci 110:1074-1083.

Sarter M, Bodewitz G, Stephens DN (1988) Attenuation of scopolamine-induced impairment of spontaneous alternation behaviour by antagonist but not inverse agonist and agonist $\beta$-carbolines. Psychopharmacology 94:491-495.

Shen J, Barnes CA, Wenk GL, McNaughton BL (1996) Differential effects of selective immunotoxic lesions of medial septal cholinergic cells on spatial working and reference memory. Behav Neurosci 110:1181-1186.

Squire LR, Zola-Morgan S (1991) The medial temporal lobe memory system. Science 253:1380-1386.

Wan RQ, Pang K, Olton DS (1994) Hippocampal and amygdaloid involvement in nonspatial and spatial working memory in rats: effects of delay and interference. Behav Neurosci 108:866-882.

Wan RQ, Givens BS, Olton DS (1995) Opioid modulation of working memory: intraseptal, but not intraamygdaloid, infusions of $\beta$-endorphin impair performance in spatial alternation. Neurobiol Learn Mem 63: $74-86$. 